\title{
Virtual Screening of 1,4-Naphthoquinone Derivatives for Inhibition of a Key Cancer Signaling Protein, AKT1 Kinase
}

\author{
MOHD REHAN $^{1,2}$ and MAGED MOSTAFA ${ }^{1,2,3}$ \\ ${ }^{1}$ King Fahd Medical Research Center, King Abdulaziz University, Jeddah, Kingdom of Saudi Arabia; \\ ${ }^{2}$ Department of Medical Laboratory Technology, Faculty of Applied Medical Sciences, \\ King Abdulaziz University, Jeddah, Kingdom of Saudi Arabia; \\ ${ }^{3}$ Molecular Genetics and Enzymology Department, Human Genetics Division and Genome Research, \\ National Research Centre, Cairo, Egypt
}

\begin{abstract}
Background/Aim: AKT, also known as protein kinase $B(P K B)$, is an established therapeutic target in cancer and its inhibitors are increasingly designed. The anticancer potential of a compound class naphthoquinones has been constantly realized. The current work aimed to explore AKT1 inhibitors from 1,4-naphthoquionone derivatives. Materials and Methods: A library of 1,4-naphthoquionone derivatives was formed using similarity search and visual analysis. The library was used for virtual screening using molecular docking. For the screened compounds, the detailed binding pose analysis, binding energy and dissociation constant calculations were performed. Results: The top 10 screened compounds were proposed as potential AKT1 inhibitors with anti-cancer activity. The compounds were checked for any reported activity, and our 2nd rank compound was reported to have anti-cancer activity. Conclusion: Our study proposes 10 compounds as potential AKT1 inhibitors and anticancer agents and also provides insights into their binding. This study also proposes AKT1 as a potential target of the reported anticancer compound, CID: 341807 .
\end{abstract}

Cancer is notably a serious medical condition causing disease and deaths worldwide and presents a major challenge to the scientific community. Over the past fifty years many groups are trying to discover effective targeted therapies, which involve designing of inhibitors against one or more proteins of a target signaling pathway $(1,2)$. One of the most frequently targeted pathways in cancer is the PI3K/AKT/mTOR pathway,

Correspondence to: Mohd Rehan, King Fahd Medical Research Center, King Abdulaziz University, Jeddah, Kingdom of Saudi Arabia. Tel: +966 531368289, e-mail: mrehan786@gmail.com

Key Words: Naphthoquinone, AKT, PKB, inhibitor, protein kinase, anti-cancer, virtual screening. which is crucial for cell growth, proliferation, and survival. The pathway is tightly regulated inside the normal cell, however, increased activity of this pathway is often observed in various human cancers, including breast, lung, endometrial, and prostate cancer (3-7). AKT, also known as protein kinase B (PKB), is a member of serine/threonine AGC protein kinase family. AKT kinase in mammals comprises three isoforms: i) Akt1/PKB $\alpha$, ii) $A k t 2 / \mathrm{PKB} \beta$ and iii) $\mathrm{Akt} 3 / \mathrm{PKB} \gamma$ that are expressed by distinct genes. The three isoforms are highly homologous sharing a similar domain structure containing $\mathrm{N}$-terminal pleckstrin homology $(\mathrm{PH})$ domain, a central kinase domain, and a carboxy-terminal regulatory domain. AKT plays role in cellular proliferation, differentiation, and survival $(8,9)$. Elevated activation of all three AKT isoforms has been observed in various human cancers (10-17). AKT1 $1^{\mathrm{E} 17 \mathrm{~K}}$ mutations are most frequently found in breast, bladder, cervix and prostate cancers, while increased activity of AKT has been shown to cause resistance to chemotherapy and radiotherapy (18-22). This link of AKT activation and cancer made AKT an attractive therapeutic target for anti-cancer therapy (23). Recent studies have shown that the chemical inhibitors of AKT can successfully overcome chemotherapy resistance when used alone or in combination with standard chemotherapy (24-26). AKT inhibitors are mainly grouped into two classes: i) allosteric inhibitors and ii) ATP-competitive inhibitors/catalytic inhibitors. Allosteric inhibitors of AKT bind to the Pleckstrin homology (PH) domain and prevent membrane localization, leading to blocking of AKT phosphorylation and activation. On the other hand, ATP competitive inhibitors/catalytic inhibitors prevent the catalytic/phosphorylation function of the protein. Various ATP competitive inhibitors of AKT are in different phases of clinical trials concerning cancer treatment $(27,28)$.

Anticancer activity involving the apoptotic role of AKT has been well reported for the 1,4 naphthoquinones (29-31). The derivatives of 1,4 napthoquinone, also called naphthoquinones, form one of the most important and widely distributed chemical class comprised of diverse set of plant 
metabolites (32). The naphthoquinones have shown biological activities against fungi, pathogenic protozoa, as well as cancer cells (33-42).

Designing novel inhibitors has now been made easy using computational methods, such as virtual screening through molecular docking. Virtual screening is the process of screening compound libraries for their potential to bind specific sites on the target protein. Computational methods, including molecular modeling, docking, and binding simulations have been extensively used for the prediction of better drug candidates (43-50). This study aimed to retrieve existing naphthoquinones, as derivatives of 1,4-naphthoquionone, and to form a chemical library. This was followed by a virtual screening of this library against the catalytic site/ATP binding site of AKT1 for ATP competitive inhibitors. Finally, the study proposed ten naphthoquinone derivatives with the highest score as potential ATP competitive inhibitors of AKT1, which may provide effective anti-cancer activity.

\section{Materials and Methods}

Data retrieval. The 3-D structure of AKT1 was retrieved from protein data bank (PDB) with PDB Id: 3 mvh and the 3-D structures of chemical compounds were obtained from PubChem database (https://pubchem.ncbi.nlm.nih.gov/). The co-complex structure of AKT1 (PDB Id: 3mvh) with a bound native ATP competitive inhibitor mentioned as three letter symbol 'WFE' in PDB structure file was selected, as its presence was required for the binding of the site probe on the protein.

Molecular docking. Molecular docking of the chemical compounds to the AKT1 catalytic site was performed using Dock, version 6.5 [University of California, San Francisco (UCSF), USA] (51). The input structure files of proteins and chemical compounds were prepared for docking using Chimera version 1.6.2 (UCSF, USA) (52). The binding site for docking was selected as the area within $10 \AA$ around the bound native inhibitor.

Analyses of docked protein-ligand complex. The docked compounds in the catalytic site of AKT1 were analyzed using Chimera v.1.6.2 (52) and Pymol, version 1.3 (Schrodinger, LLC, NY, USA) (53). The ligand-protein interaction plots were generated using LigPlot+, version 2.1 [European Molecular Biology Laboratory-Europian Bioinformatics Institute (EMBL-EBI), Cambridge, UK] (54), which shows schematically non-bonding contacts and hydrogen bonding interactions. In addition to the score obtained from the molecular docking, the binding energy and dissociation constant were predicted using XScore, version 1.2.11 (University of Michigan Medical School, Ann Arbor, MI, USA) (55).

\section{Results}

Library of 1,4-naphthoquinone derivatives. 1,4-naphthoquinone (Figure 1A) with PubChem Compound Id, CID: 8530 was searched for similar compounds in PubChem and was filtered for drug-likelihood using Lipinski's rule of five (56), yielding 954 compounds. These compounds were visually analyzed for possessing a 1,4-naphthoquinone scaffold, which shortlisted 210 compounds. This library of 210 compounds of 1,4naphthoquinone derivatives or 1,4-naphthoquinones was used in the current study for virtual screening. In a previous study (57), we considered compounds similar to 1,4-naphthoquinone as naphthoquinone analogs, however, in the current study, the compounds were considered on the basis of having a common 1,4-naphthoquinone scaffold.

Virtual screening of 1,4-naphthoquinone derivatives. For the virtual screening of 1,4-naphthoquinone derivatives against human AKT1, the molecular docking of all derivatives was performed and the compounds were arranged in the order of decreasing dock scores and were plotted in a histogram (Figure 2). Finally, the top 10 scoring compounds (Figures 1B-K) were proposed as potential inhibitors of AKT1 with anti-cancer activity and were, thus, selected for further analysis.

Molecular docking analyses of top 10 screened compounds. The top 10 dock scoring 1,4-naphthoquinone derivatives were selected and proposed as potential inhibitors of AKT1. The compounds were named based on their PubChem CID. The top 10 compounds, as arranged in the order of decreasing dock score, are the following: i) '56981794', ii) '394547', iii) '14640331', iv) '341807', v) ‘5922604', vi) '1473358', vii) '14907553', viii) ‘6109815', ix) ‘18987539', and $\mathrm{x}$ ) '71652351'. The molecular docking analyses of these compounds are briefed below.

With regards to the molecular architecture of the top dock scoring compound, '56981794', bound to the catalytic site of AKT1 protein (Figure 3A), this was found to interact with 11 residues: Leu-156, Gly-157, Val-164, Ala-177, Met-227, Glu-234, Met-281, Thr-291, Phe-438, Asp-439, and Phe-442 (Figure 4A, Table I). The robust binding strength of this compound to the AKT1 catalytic site was evident from the binding score values, including i) the dock score (-40.36), ii) the binding energy $(-8.73)$, and iii) the $\mathrm{pK}_{\mathrm{d}}$ (6.40) (Table II). Similarly, the AKT1 interacting residues for the native ligand, WFE, include the following: Phe-438, Asp-292, Thr291, Met-281, Glu-234, Ala-230, Tyr-229, Glu-228, Met227, Thr-211, Lys-179, Ala-177, Val-164, Gly-162, Gly-159, and Leu-156 (Figure 4). Of the 11 interacting residues for this proposed compound, eight residues were found to be common among the interacting residues of the native ligand (Table I). This observation of blocking the same set of residues as the native inhibitor added value to the prediction of the proposed compound as inhibitor.

The top second dock scoring compound, '394547', bound to the catalytic site (Figure $3 \mathrm{~A}$ ), interacted with 12 residues, including: Leu-156, Gly-157, Lys-158, Gly-159, Gly-162, Lys-163, Val-164, Lys-179, Glu-234, Lys-276, Asp-292, and Phe-442 (Figure 4B, Table I). The high absolute values of 
Rehan and Mostafa: Exploring Naphthoquinones for Onco-Signaling Protein AKT1 Inhibition

A. Scaffold

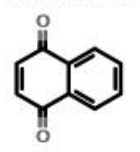

D. '14640331'

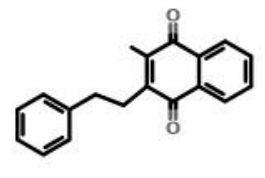

G. '1473358'

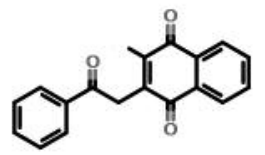

J. '18987539'

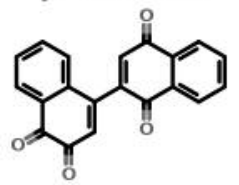

B. '56981794'

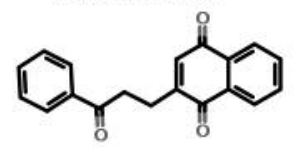

E. '341807'

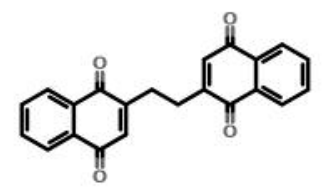

H. '14907553'

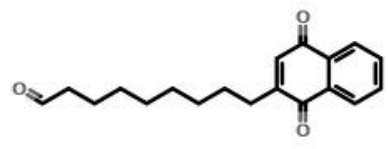

K. '71652351'

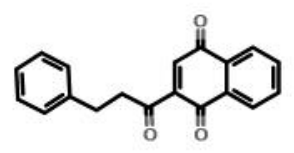

Figure 1. Two dimensional structure of common scaffold of 1,4-naphthaquinone (A) and its proposed derivatives (B-K) for AKT1 inhibition.

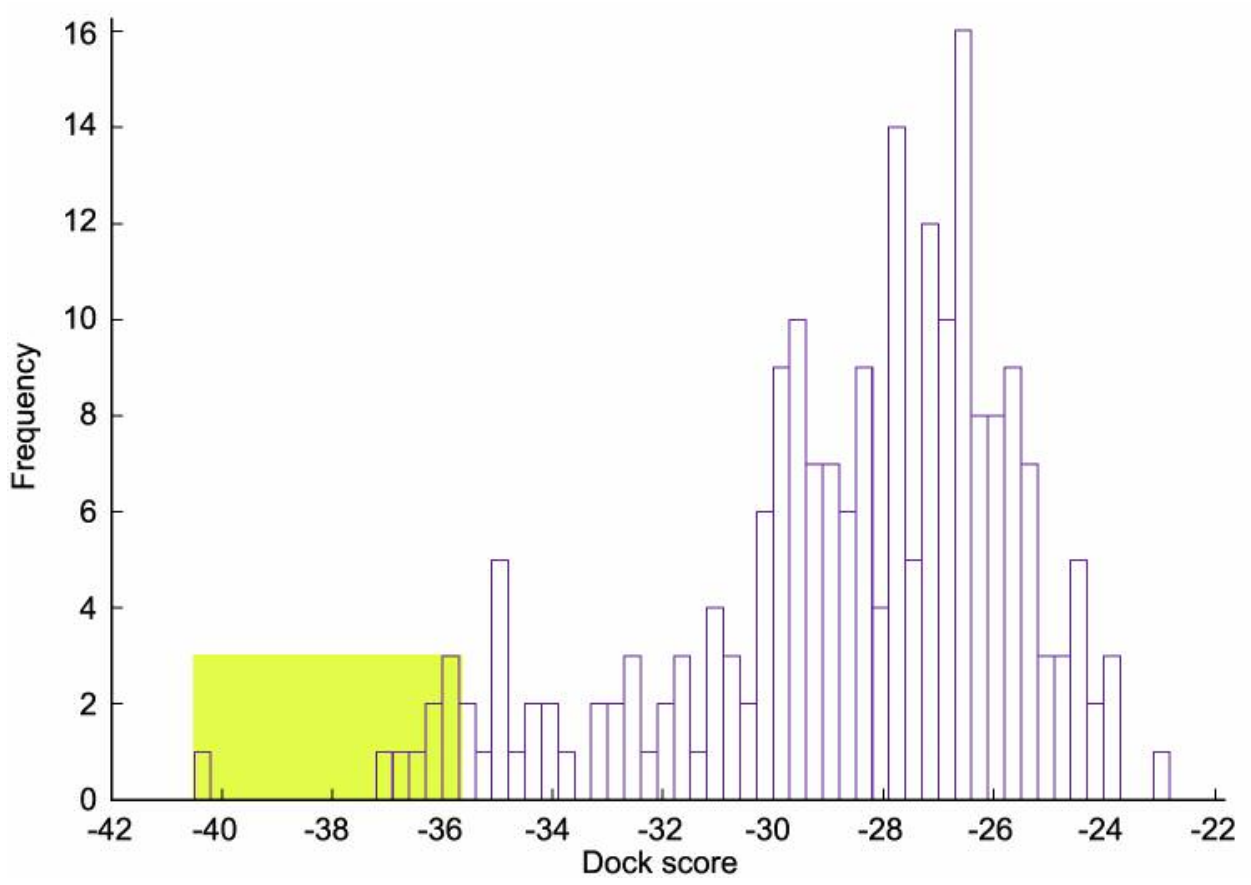

Figure 2. Histogram plot for dock scores of 1,4-naphthaquinone derivatives for molecular docking with AKT1. The extreme left region of the plot shows the cutoff that highlights the region from where the proposed AKT1 inhibitors are selected.

3825 

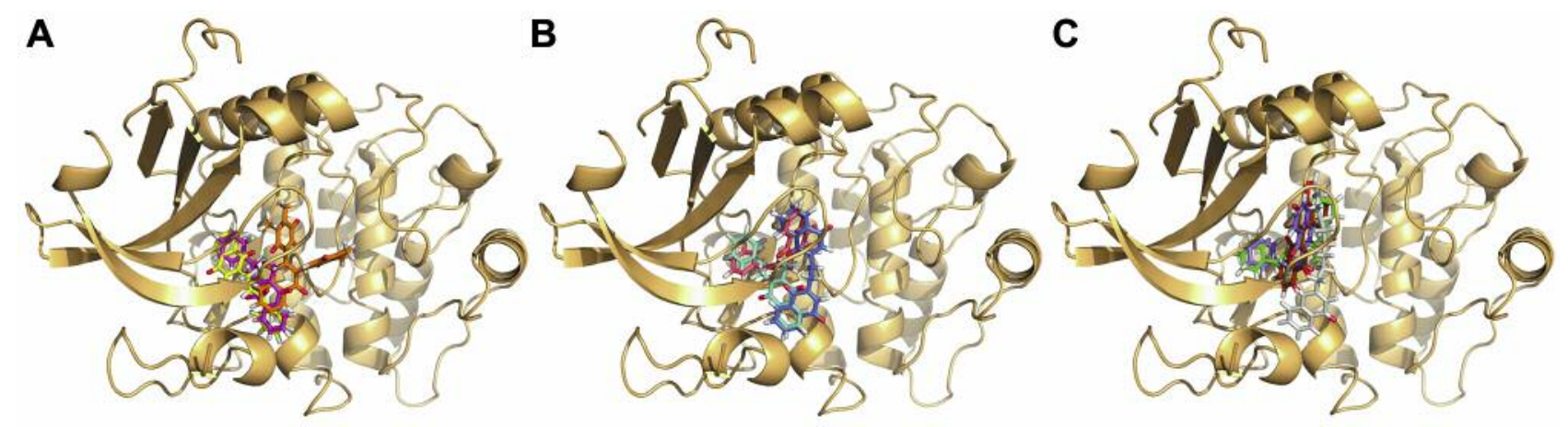

Figure 3. AKT1 with 10 proposed docked inhibitory compounds. AKT1 is shown in a cartoon representation (light orange) and the compounds are in stick representation. (A) Binding of the first three compounds: '56981794', '394547', and '14640331', are colored yellow, orange, and magenta, respectively. (B) Binding of the next three compounds: '341807', '5922604', and '1473358', are colored blue, deep salmon, and cyan, respectively. (C) Binding of the last four compounds: '14907553', '6109815', '18987539', and '71652351', are colored white, green, brown, and slate, respectively.

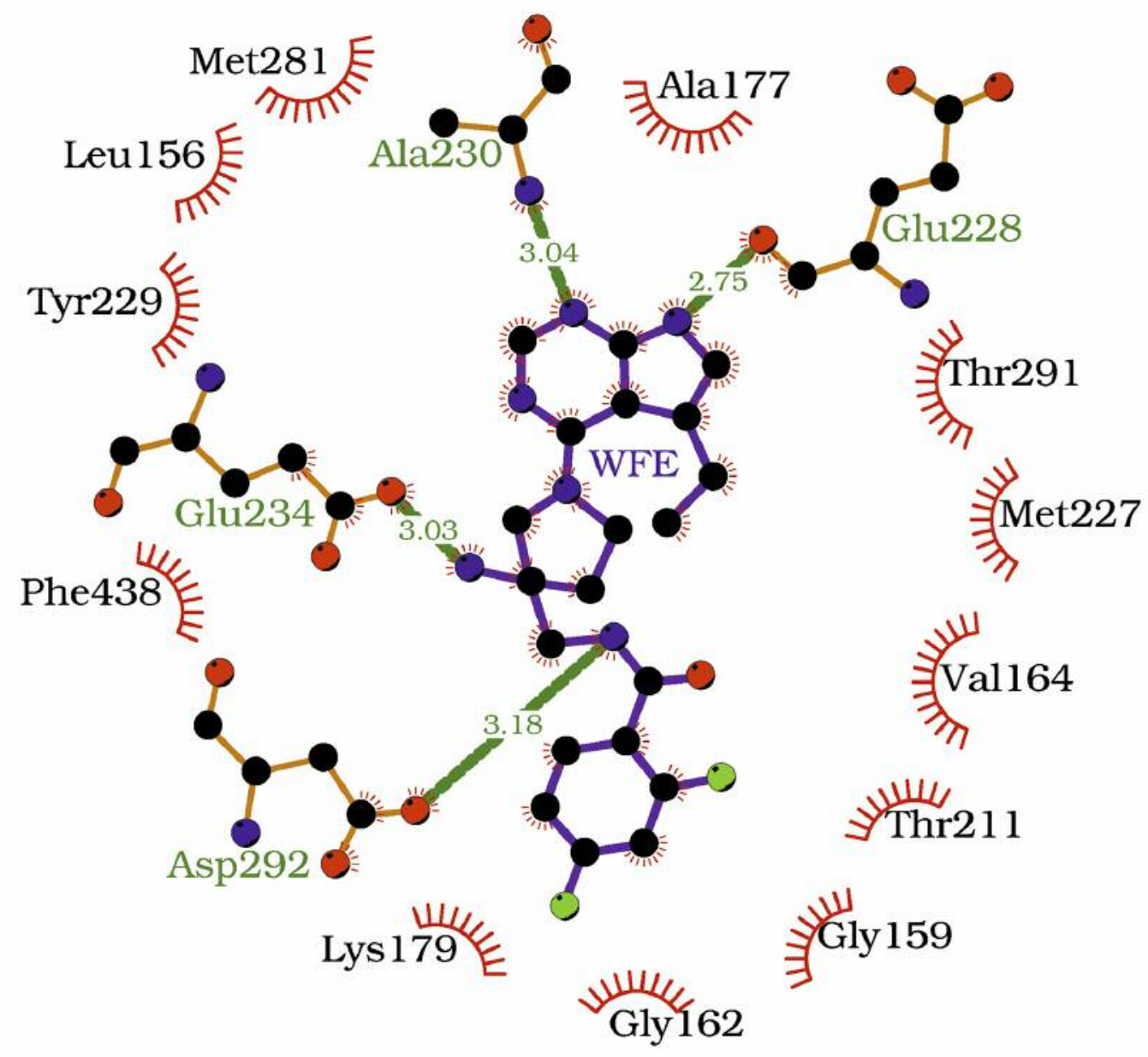

Figure 4. Protein-ligand interaction plot for AKT1 and its native inhibitor, WFE. The amino acid residues involved in non-bonded contacts are shown as red arches with bristles. The hydrogen bond interactions are shown as green dashed lines labeled with bond length (in $\AA$ ) and the residues involved in these interactions are in ball-and-stick representation. The native inhibitor, WFE is shown in ball-and-stick representation, where a ball represents an atom and a stick between two atoms represents a covalent bond. The color of the balls (representing atoms) distinguishes among atom types i.e., the black balls represent carbon atoms, blue balls represent the nitrogen atoms, green balls represent the fluorine atoms, and the red balls represent the oxygen atoms. 
Table I. Interacting residues of AKT1 for the ten proposed compounds. The compounds indicated by their PubChem CID are in the first row of the table. Each column contains interacting residues for the compound mentioned on the top of the column. The common interacting residues for different compounds are placed in the same row and the interacting residues common with those of native ligand are in bold font.

\begin{tabular}{|c|c|c|c|c|c|c|c|c|c|}
\hline 56981794 & 394547 & 14640331 & 341807 & 5922604 & 1473358 & 14907553 & 6109815 & 18987539 & 71652351 \\
\hline Leu-156 & Leu-156 & Leu-156 & Leu-156 & Leu-156 & Leu-156 & Leu-156 & - & Leu-156 & - \\
\hline Gly-157 & Gly-157 & Gly-157 & Gly-157 & Gly-157 & Gly-157 & Gly-157 & - & Gly-157 & Gly-157 \\
\hline- & Lys-158 & - & - & - & - & - & - & Lys-158 & - \\
\hline- & Gly-159 & - & Gly-159 & Gly-159 & - & - & - & - & Gly-159 \\
\hline- & - & - & - & - & - & Phe-161 & - & Phe-161 & - \\
\hline- & Gly-162 & - & - & Gly-162 & - & - & - & Gly-162 & Gly-162 \\
\hline- & Lys-163 & - & - & - & - & - & - & - & - \\
\hline Val-164 & Val-164 & Val-164 & - & Val-164 & Val-164 & - & Val-164 & Val-164 & Val-164 \\
\hline Ala-177 & - & Ala-177 & - & Ala-177 & Ala-177 & - & Ala-177 & - & - \\
\hline- & Lys-179 & - & - & - & - & Lys-179 & Lys-179 & Lys-179 & Lys-179 \\
\hline- & - & - & - & - & - & Leu-181 & - & Leu-181 & Leu-181 \\
\hline Met-227 & - & - & - & - & - & - & - & - & - \\
\hline Glu-234 & Glu-234 & Glu-234 & Glu-234 & Glu-234 & Glu-234 & Glu-234 & Glu-234 & Glu-234 & Glu-234 \\
\hline- & - & - & Phe-236 & - & - & Phe-236 & - & - & - \\
\hline- & Lys-276 & - & - & - & - & - & - & - & - \\
\hline- & - & - & - & - & - & - & Glu-278 & - & - \\
\hline- & - & - & - & Asn-279 & - & - & - & - & - \\
\hline Met-281 & - & Met-281 & - & Met-281 & Met-281 & - & Met-281 & - & Met-281 \\
\hline Thr-291 & - & - & - & Thr-291 & - & - & Thr-291 & - & - \\
\hline- & Asp-292 & Asp-292 & - & Asp-292 & - & - & Asp-292 & Asp-292 & Asp-292 \\
\hline Phe-438 & - & Phe-438 & - & - & Phe-438 & - & - & - & - \\
\hline Asp-439 & - & Asp-439 & Asp-439 & - & Asp-439 & Asp-439 & - & - & - \\
\hline Phe-442 & Phe- 442 & Phe- 442 & Phe- 442 & - & Phe- 442 & Phe- 442 & - & - & - \\
\hline
\end{tabular}

the binding strength scores, dock score $(-36.91)$, binding energy (-7.93), and $\mathrm{pK}_{\mathrm{d}}(5.82)$, indicate the binding quality required for good inhibition (Table II). Of the 12 interacting residues, seven residues were found common with residues of the native ligand (Table I). This indicates that the proposed compound is also blocking part of the same set of residues as those of the native inhibitor and thus, inhibits the protein in a similar way.

The third top dock scoring compound, '14640331', bound to the catalytic site (Figure 3A), interacted with the following 10 residues: Leu-156, Gly-157, Val-164, Ala-177, Glu-234, Met281, Asp-292, Phe-438, Asp-439, and Phe-442 (Figure 5C, Table I). The high absolute values of binding strength scores, such as dock score (-36.79), binding energy $(-8.25)$, and $\mathrm{pK}_{\mathrm{d}}$ (6.05) show a quality binding required for adequate inhibition (Table II). Of the 10 interacting residues, seven residues were common with the interacting residues of the native ligand (Table I). This indicates that the proposed compound also interacted to an extent with the same set of residues as the ones of the native inhibitor and thus, inhibits the protein in a similar way.

The fourth top docking score compound, '341807', binding to the catalytic site (Figure 3B) interacted with seven residues: Leu-156, Gly-157, Gly-159, Glu-234, Phe-236, Asp-439, and Phe-442 (Figure 5D, Table I). The high absolute values of binding strength scores, dock score
Table II. The ten proposed compounds and binding scores. The compounds indicated with PubChem CIDs are provided with Dock score, binding energy, and $p K_{d}$ values.

\begin{tabular}{lccc}
\hline Compound & Dock score & Binding energy & $\mathrm{pK}_{\mathrm{d}}$ \\
\hline 56981794 & -40.36 & -8.73 & 6.40 \\
394547 & -36.91 & -7.93 & 5.82 \\
14640331 & -36.79 & -8.25 & 6.05 \\
341807 & -36.32 & -7.81 & 5.73 \\
5922604 & -36.07 & -8.11 & 5.95 \\
1473358 & -36.01 & -8.40 & 6.16 \\
14907553 & -35.99 & -6.79 & 4.98 \\
6109815 & -35.90 & -8.10 & 5.94 \\
18987539 & -35.73 & -7.72 & 5.66 \\
71652351 & -35.65 & -7.85 & 5.75 \\
\hline
\end{tabular}

(-36.32), binding energy (-7.81), and $\mathrm{pK}_{\mathrm{d}}$ (5.73) were also indicative of a quality binding required for adequate inhibition (Table II). Of the seven interacting residues of ' 341807 ', three residues were found common with the interacting residues of the native ligand (Table I).

The fifth top docking score compound, '5922604', bound to the catalytic site (Figure 3B) was found to interact with 11 residues: Leu-156, Gly-157, Gly-159, Gly-162, Val-164, Ala- 


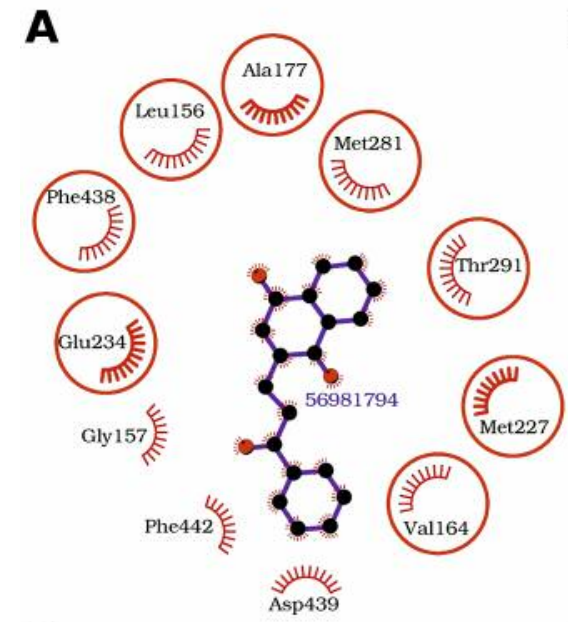

D

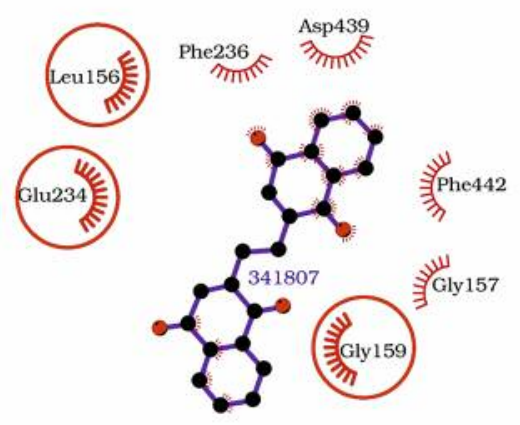

B

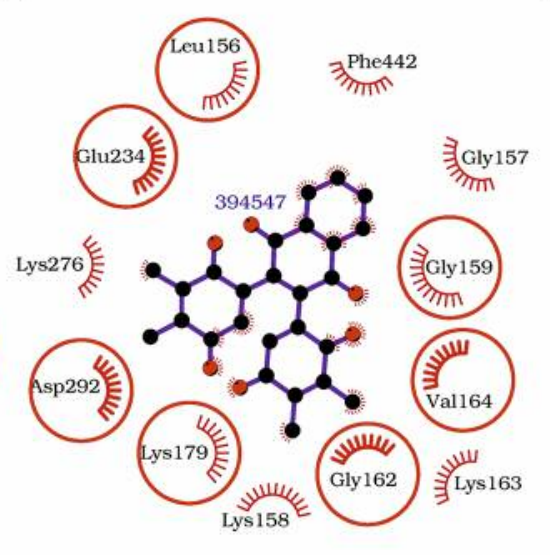

E

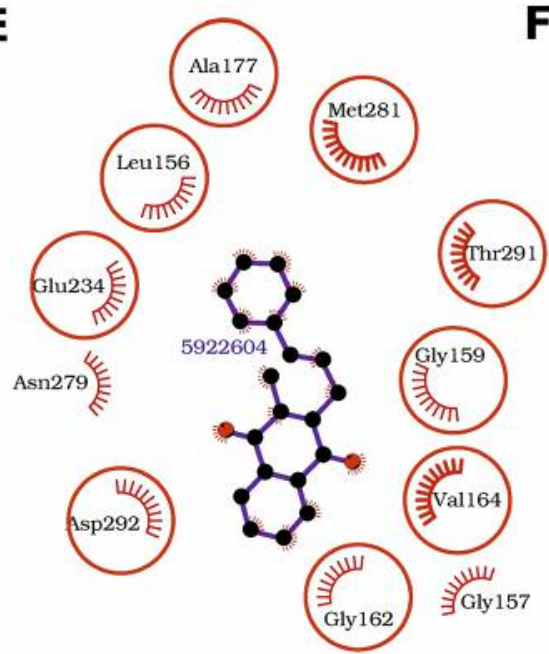

C

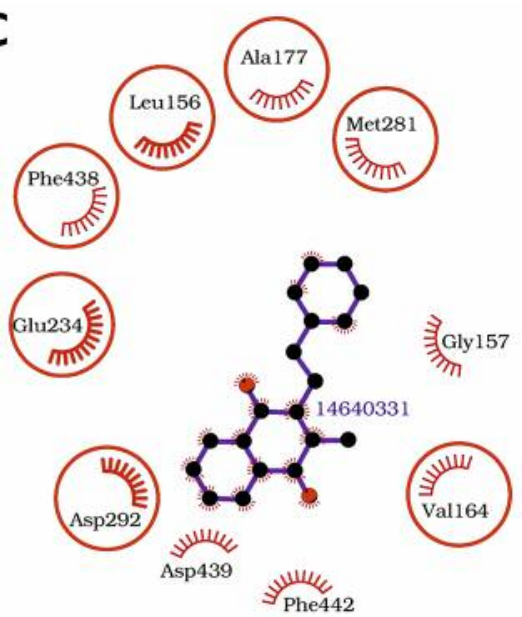

$\mathbf{F}$

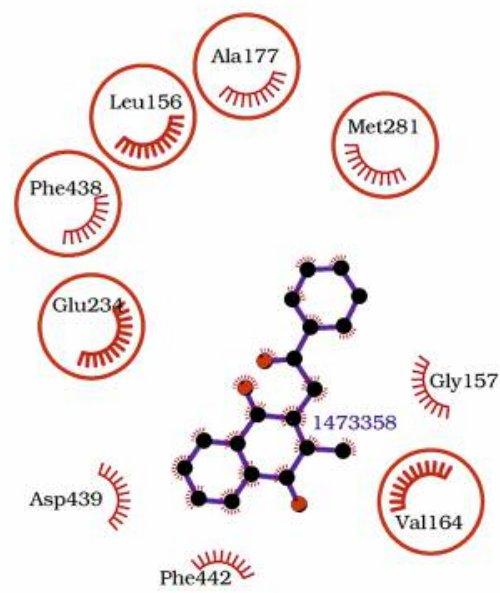

Figure 5. Protein-ligand interaction plots for AKT1 and its proposed inhibitors. The amino acid residues involved in non-bonded contacts are shown as red arches with bristles. The interacting residues common with those of the native inhibitor are encircled. The proposed inhibitors are shown in ball-and-stick representations, where a ball represents an atom and a stick between two atoms represents a covalent bond. The color of the balls (representing atoms) distinguishes between carbon (black) and oxygen (red) atoms.

177, Glu-234, Asn-279, Met-281, Thr-291, and Asp-292 (Figure $5 \mathrm{E}$, Table I). The absolute values of the binding strength scores, dock score (-36.07), binding energy $(-8.11)$, and $\mathrm{pK}_{\mathrm{d}}(5.95)$ were good enough for adequate inhibition (Table II). Of the 11 interacting residues, nine residues were found common with the interacting residues of the native ligand (Table I).

The sixth docking score compound, ' $1473358^{\prime}$, bound to the catalytic site (Figure 3B), interacted with nine residues: Leu-156, Gly-157, Val-164, Ala-177, Glu-234, Met-281, Phe-438, Asp-439, and Phe-442 (Figure 5F, Table I). The high absolute values of dock score $(-36.01)$, binding energy $(-8.40)$, and $\mathrm{pK}_{\mathrm{d}}$ (6.16) indicated a good binding quality required for adequate inhibition (Table II). Of the nine interacting residues, six residues were found common with the interacting residues of the native ligand (Table I).
The seventh dock score compound, '14907553', bound to the catalytic site (Figure 3C) interacted with nine residues: Leu-156, Gly-157, Phe-161, Lys-179, Leu-181, Glu-234, Phe-236, Asp-439, and Phe-442 (Figure 6A, Table I). The binding strength of the compound is reasonably good as evidenced from high absolute values of dock score $(-35.99)$, binding energy (-6.79), and $\mathrm{pK}_{\mathrm{d}}$ (4.98) (Table II). Of the nine interacting residues, three residues were shared with the interacting residues of the native ligand (Table I).

The eighth docking score compound, ' 6109815 ', bound to the catalytic site (Figure 3C) interacted with eight residues: Val-164, Ala-177, Lys-179, Glu-234, Glu-278, Met-281, Thr291, and Asp-292 (Figure 6B, Table I). The absolute values of the binding strength scores: dock score (-35.90), binding energy (-8.10), and $\mathrm{pK}_{\mathrm{d}}$ (5.94), were reasonably high to 
A

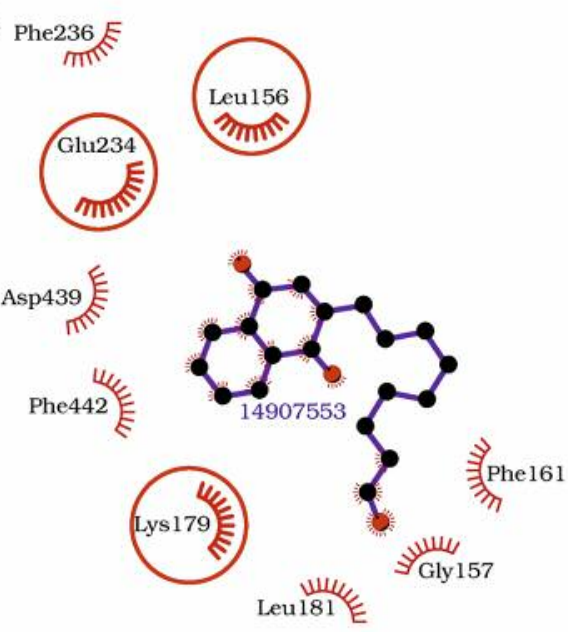

C

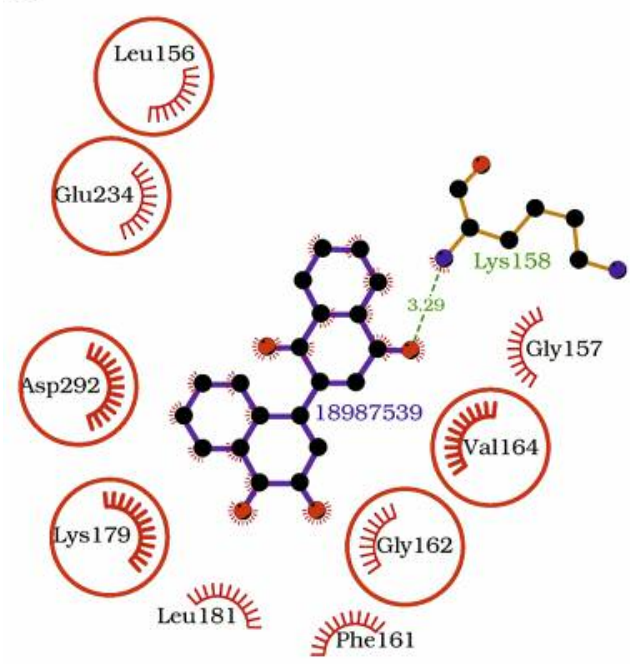

B
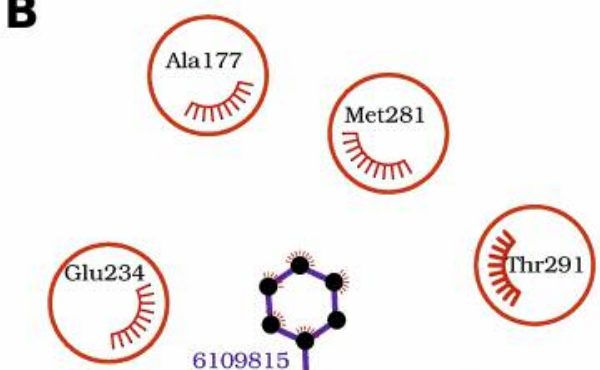
6109815
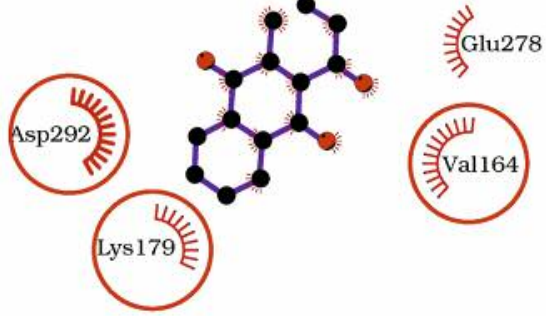

D

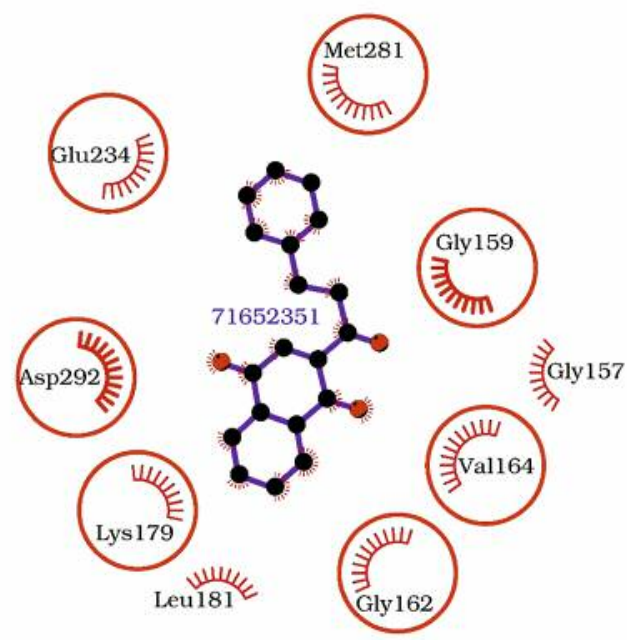

Figure 6. Protein-ligand interaction plots for AKT1 and its proposed inhibitors. The amino acid residues involved in non-bonded contacts are shown as red arches with bristles. The interacting residues common with those of the native inhibitor are encircled. The hydrogen bond interactions are shown as a green dashed line labeled with the bond length (in $\AA$ ) and the residues involved in hydrogen bonds are in ball-and-stick representation. The proposed inhibitors are also shown in ball-and-stick representations, where a ball represents an atom and a stick between two atoms represents a covalent bond. The color of the balls (representing atoms) distinguishes between carbon (black) and oxygen (red) atoms.

satisfy the criteria for adequate inhibition (Table II). Of the eight interacting residues, seven residues were shared with the list of interacting residues of the native ligand (Table I).

The nineth docking score compound, '18987539', bound to the catalytic site (Figure 3C), interacted with 10 residues: Leu-156, Gly-157, Lys-158, Phe-161, Gly-162, Val-164, Lys179, Leu-181, Glu-234, and Asp-292 (Figure 6C, Table I). The binding strength score values: dock score $(-35.73)$, binding energy (-7.72), and $\mathrm{pK}_{\mathrm{d}}$ (5.66), were indicative of good binding required for adequate inhibition (Table II). In addition to the non-bonding interactions, the compound was also found to be stabilized by a hydrogen bond involving residue Lys-158. Of the 10 interacting residues, six residues were shared with the list of interacting residues of the native ligand (Table I).

Finally, the last selected compound (10th rank), '71652351', bound to the catalytic site (Figure 3C), interacted with nine residues: Gly-157, Gly-159, Gly-162, Val-164, Lys-179, Leu-181, Glu-234, Met-281, and Asp292 (Figure 6D, Table I). The high absolute values of dock 
score $(-35.65)$, binding energy $(-7.85)$, and $\mathrm{pK}_{\mathrm{d}}(5.75)$ indicated a binding quality required for good inhibition (Table II). Of the nine interacting residues, seven residues were share with the list of interacting residues of the native ligand (Table I).

Taken together, the top ten compounds selected shared many of the interacting residues with the native ligand, thereby suggesting that they could have an inhibitory ability, possibly similar to that of the native ligand.

\section{Discussion}

In the current study we retrieved all known 1,4naphthaquinone derivatives from PubChem and formed a library of them. Virtual screening of the compound library led us to propose the top 10 ranking compounds as potential inhibitors. The compounds were named after the PubChem ID, CID, arranged in the order of AKT1 inhibiting potential. When these compounds were looked up in the PubChem bioassay database (58) for possessing any type of activity in bioassays, six compounds '394547', '341807', '5922604', '1473358', '6109815', and '71652351' were found active/inactive for one or another activity. The compound '394547' (2nd rank) has been exhaustively tested in 61 different cancer cell lines using the National Cancer Institute (NCI) human tumor cell line growth inhibition assay and it has been found active in the following four cancer cell lines: i) MALME-3M melanoma cell line, ii) COLO 205 colon cell line, iii) CCRF-CEM leukemia cell line, and iv) HL-60(TB) leukemia cell line, as reported in the PubChem bioassay AID: 29, 67, 119, and 125 (59-62). This finding of the 2nd rank compound, '394547', possessing anti-cancer properties added weight to our screening of potential AKT1 inhibitors and anti-cancer compounds. Another compound, '341807', (4th rank) has been found inactive for the NCI Yeast anticancer drug screening reported in PubChem bioassay AID: 155, 157, 161, 165, 167, and 175, where anti-cancer activity is measured as the growth inhibition of yeast strains with defined genetic alterations mimicking a cancer cell model (63-68). Also, this compound has been found inactive for the NCI AIDS antiviral assay reported in the PubChem bioassay AID 179 (69) and the NCI In Vivo Anticancer Drug Screen for the tumor model P388 of Leukemia (intraperitoneal) in CD2F1 (CDF1) mice reported in the PubChem bioassay AID 330 (70). Another compound, '5922604', (5th rank) has been found inactive for the NCI AIDS antiviral assay reported in the PubChem bioassay AID 179 (69), however, anti-cancer activity is not reported. The next compound, '1473358', (6th rank) has been found active against multiple targets reported in the PubChem bioassay results for CID 1473358 and inactive against AKT1 in the HTS assay for inhibitors of AKT phosphorylation reported in the PubChem bioassay AID 651550 (71). Another compound, '6109815', (8th rank) has been found inactive in the NCI In Vivo anticancer drug screening for the tumor model P388 of Leukemia (intraperitoneal) in CD2F1 (CDF1) mice reported in the PubChem bioassay AID 330 (70). Finally, the compound '71652351' (10th rank) has been reported to have unspecified activity on WHCO1 cancer cell lines reported in the PubChem bioassay AID 735529 (72).

Interestingly, the second rank proposed compound, '394547', has been reported to exhibit anticancer properties (59-62), however, the molecular target for this compound is not reported. In the current study, this compound was picked as a potential inhibitor of AKT1 kinase. Therefore, this study also explored the molecular target of the anticancer compound with CID: 394547 and predicted AKT1 as a potential molecular target.

When the 10 lowest scoring naphthoquinones were checked in PubChem bioassay database for any reported anti-cancer activity, only one compound with CID 345400 was found to be inactive for all six NCI yeast anticancer drug screenings reported in PubChem bioassays AID: 155, 157, 161, 165, 167, and 175 (63-68). This added extra weight to our predictions as this was in agreement with our expectations that the high scoring compounds should be active while the low scoring compounds should be inactive in terms of anti-cancer activity.

Thus, the detailed analyses of the docked complexes of top 10 dock scoring compounds revealed their respective interacting residues, binding strength scores, and molecular interactions. Finally, the binding of the selected compounds were also compared to the binding of the native inhibitor. This comparison revealed that the proposed compounds were also interacting with a large part of the same set of residues as those of the native inhibitor and, thus, they may be inhibiting AKT1 in a similar way. The findings of this screening for potential inhibitors of AKT1 with anti-cancer properties will hopefully be an attractive opportunity for experimental biologists to test these in the laboratory.

\section{Conflicts of Interest}

None.

\section{Authors' Contributions}

MR conceived and designed the experiments and analyzed the data. MR and MM performed the experiments and wrote the paper.

\section{Acknowledgements}

This work was funded by the Deanship of Scientific Research (DSR), King Abdulaziz University, Jeddah, under grant No: (G:463141-37). The authors, therefore, acknowledge with thanks DSR technical and financial support. 


\section{References}

1 Sawyers C: Targeted cancer therapy. Nature 432(7015): 294-297, 2004. PMID: 15549090. DOI: 10.1038/nature03095

2 Baudino TA: Targeted cancer therapy: The next generation of cancer treatment. Curr Drug Discov Technol 12(1): 3-20, 2015. PMID: 26033233.

3 Myers AP and Cantley LC: Targeting a common collaborator in cancer development. Sci Transl Med 2(48): 48ps45, 2010. PMID: 20826838. DOI: 10.1126/scitranslmed.3001251

4 Sarris EG, Saif MW and Syrigos KN: The biological role of pi3k pathway in lung cancer. Pharmaceuticals 5(11): 1236-1264, 2012. PMID: 24281308. DOI: $10.3390 /$ ph5111236

5 Morgan TM, Koreckij TD and Corey E: Targeted therapy for advanced prostate cancer: Inhibition of the pi3k/akt/mtor pathway. Curr Cancer Drug Targets 9(2): 237-249, 2009. PMID: 19275762.

6 Liu H, Zhang L, Zhang X and Cui Z: Pi3k/AKT/MTOR pathway promotes progestin resistance in endometrial cancer cells by inhibition of autophagy. Onco Targets Ther 10: 2865-2871, 2017. PMID: 28652768. DOI: 10.2147/OTT.S95267

7 Shuttleworth SJ, Silva FA, Cecil ARL, Tomassi CD, Hill TJ, Raynaud FI, Clarke PA and Workman P: Progress in the preclinical discovery and clinical development of class $\mathrm{i}$ and dual class i/iv phosphoinositide 3-kinase (pi3k) inhibitors. Curr Med Chem 18(18): 2686-2714, 2011. PMID: 21649578.

8 Manning BD and Toker A: AKT/PKB Signaling: Navigating the Network. Cell 169(3): 381-405, 2017. PMID: 28431241. DOI: 10.1016/j.cell.2017.04.001

9 Manning BD and Cantley LC: AKT/PKB signaling: Navigating downstream. Cell 129(7): 1261-1274, 2007. PMID: 17604717. DOI: $10.1016 /$ j.cell.2007.06.009

10 Yuan ZQ, Sun M, Feldman RI, Wang G, Ma X, Jiang C, Coppola D, Nicosia SV and Cheng JQ: Frequent activation of akt2 and induction of apoptosis by inhibition of phosphoinositide-3-oh kinase/akt pathway in human ovarian cancer. Oncogene 19(19): 2324-2330, 2000. PMID: 10822383. DOI: 10.1038/sj.onc.1203598

11 Sun M, Paciga JE, Feldman RI, Yuan Z, Coppola D, Lu YY, Shelley SA, Nicosia SV and Cheng JQ: Phosphatidylinositol-3oh kinase (pi3k)/akt2, activated in breast cancer, regulates and is induced by estrogen receptor alpha (eralpha) via interaction between eralpha and pi3k. Cancer Res 61(16): 5985-5991, 2001. PMID: 11507039.

12 Sun M, Wang G, Paciga JE, Feldman RI, Yuan ZQ, Ma XL, Shelley SA, Jove R, Tsichlis PN, Nicosia SV and Cheng JQ: Akt $1 /$ pkbalpha kinase is frequently elevated in human cancers and its constitutive activation is required for oncogenic transformation in nih3t3 cells. Am J Pathol 159(2): 431-437, 2001. PMID: 11485901.

13 Altomare DA, Tanno S, De Rienzo A, Klein-Szanto AJ, Tanno S, Skele KL, Hoffman JP and Testa JR: Frequent activation of akt2 kinase in human pancreatic carcinomas. J Cell Biochem 87(4): 470-476, 2002. PMID: 14735903.

14 Altomare DA, Wang HQ, Skele KL, De Rienzo A, Klein-Szanto AJ, Godwin AK and Testa JR: Akt and mtor phosphorylation is frequently detected in ovarian cancer and can be targeted to disrupt ovarian tumor cell growth. Oncogene 23(34): 5853-5857, 2004. PMID: 15208673. DOI: 10.1038/sj.onc.1207721.

15 Altomare DA, You H, Xiao G-H, Ramos-Nino ME, Skele KL, De Rienzo A, Jhanwar SC, Mossman BT, Kane AB and Testa
JR: Human and mouse mesotheliomas exhibit elevated akt/pkb activity, which can be targeted pharmacologically to inhibit tumor cell growth. Oncogene 24(40): 6080-6089, 2005. PMID: 15897870. DOI: $10.1038 /$ sj.onc. 1208744

16 Altomare DA and Testa JR: Perturbations of the akt signaling pathway in human cancer. Oncogene 24(50): 7455-7464, 2005. PMID: 16288292. DOI: 10.1038/sj.onc.1209085

17 Balsara BR, Pei J, Mitsuuchi Y, Page R, Klein-Szanto A, Wang $\mathrm{H}$, Unger $\mathrm{M}$ and Testa JR: Frequent activation of akt in nonsmall cell lung carcinomas and preneoplastic bronchial lesions. Carcinogenesis 25(11): 2053-2059, 2004. PMID: 15240509. DOI: $10.1093 /$ carcin/bgh226

18 Cheng JQ, Jiang X, Fraser M, Li M, Dan HC, Sun M and Tsang BK: Role of $\mathrm{x}$-linked inhibitor of apoptosis protein in chemoresistance in ovarian cancer: Possible involvement of the phosphoinositide-3 kinase/akt pathway. Drug Resist Updat 5(34): 131-146, 2002. PMID: 12237081.

19 Arlt A, Gehrz A, Müerköster S, Vorndamm J, Kruse M-L, Fölsch UR and Schäfer H: Role of nf-kappab and akt/pi3k in the resistance of pancreatic carcinoma cell lines against gemcitabine-induced cell death. Oncogene 22(21): 3243-3251, 2003. PMID: 12761494. DOI: 10.1038/sj.onc.1206390

20 Knuefermann C, Lu Y, Liu B, Jin W, Liang K, Wu L, Schmidt M, Mills GB, Mendelsohn J and Fan Z: Her2/pi-3k/akt activation leads to a multidrug resistance in human breast adenocarcinoma cells. Oncogene 22(21): 3205-3212, 2003. PMID: 12761490. DOI: 10.1038/sj.onc.1206394

21 Yuan Z-Q, Feldman RI, Sussman GE, Coppola D, Nicosia SV and Cheng JQ: Akt2 inhibition of cisplatin-induced jnk/p38 and bax activation by phosphorylation of ask1: Implication of akt2 in chemoresistance. J Biol Chem 278(26): 23432-23440, 2003. PMID: 12697749. DOI: 10.1074/jbc.M302674200

22 Nagata Y, Lan K-H, Zhou X, Tan M, Esteva FJ, Sahin AA, Klos KS, Li P, Monia BP, Nguyen NT, Hortobagyi GN, Hung M-C and $\mathrm{Yu}$ D: Pten activation contributes to tumor inhibition by trastuzumab, and loss of pten predicts trastuzumab resistance in patients. Cancer Cell 6(2): 117-127, 2004. PMID: 15324695. DOI: $10.1016 /$ j.ccr.2004.06.022

23 Cheng JQ, Lindsley CW, Cheng GZ, Yang $\mathrm{H}$ and Nicosia SV: The akt/pkb pathway: Molecular target for cancer drug discovery. Oncogene 24(50): 7482-7492, 2005. PMID: 16288295. DOI: $10.1038 /$ sj.onc. 1209088

24 Brown JS and Banerji U: Maximising the potential of akt inhibitors as anti-cancer treatments. Pharmacol Ther 172: 101-115, 2017. PMID: 27919797. DOI: 10.1016/j.pharmthera. 2016.12.001

25 Wang P, Zhang L, Hao Q and Zhao G: Developments in selective small molecule atp-targeting the serine/threonine kinase akt/pkb. Mini Revn Med Chem 11(13): 1093-1107, 2011. PMID: 22353219.

26 Polak R and Buitenhuis M: The PI3K/PKB signaling module as key regulator of hematopoiesis: Implications for therapeutic strategies in leukemia. Blood 119(4): 911-923, 2012. PMID: 22065598. DOI: 10.1182/blood-2011-07-366203

27 Nitulescu GM, Margina D, Juzenas P, Peng Q, Olaru OT, Saloustros E, Fenga C, Spandidos DA, Libra M and Tsatsakis AM: Akt inhibitors in cancer treatment: The long journey from drug discovery to clinical use (review). Int J Oncol 48(3): 869885, 2016. PMID: 26698230. DOI: 10.3892/ijo.2015.3306

28 Martelli AM, Evangelisti C, Chappell W, Abrams SL, Bäsecke J, Stivala F, Donia M, Fagone P, Nicoletti F, Libra M, Ruvolo V, 
Ruvolo P, Kempf CR, Steelman LS and McCubrey JA: Targeting the translational apparatus to improve leukemia therapy: Roles of the pi3k/pten/akt/mtor pathway. Leukemia 25(7): 1064-1079, 2011. PMID: 21436840. DOI: 10.1038/leu.2011.46

29 Tandon VK, Chhor RB, Singh RV, Rai S and Yadav DB: Design, synthesis and evaluation of novel 1,4-naphthoquinone derivatives as antifungal and anticancer agents. Bioorg Med Chem Lett 14(5): 1079-1083, 2004. PMID: 14980639. DOI: 10.1016/j.bmcl.2004.01.002

30 Yuk DY, Ryu CK, Hong JT, Chung KH, Kang WS, Kim Y, Yoo HS, Lee MK, Lee CK and Yun YP: Antithrombotic and antiplatelet activities of 2-chloro-3-[4-(ethylcarboxy)-phenyl]amino-1,4-naphthoquinone (nq12), a newly synthesized 1,4naphthoquinone derivative. Biochem Pharmacol 60(7): 10011008, 2000. PMID: 10974210.

31 Ravelo AG, Estévez-Braun A, Chávez-Orellana H, Pérez-Sacau E and Mesa-Siverio D: Recent studies on natural products as anticancer agents. Curr Top Med Chem 4(2): 241-265, 2004. PMID: 14754457.

32 Babula P, Adam V, Havel L and Kizek R: Noteworthy secondary metabolites naphthoquinones - their occurrence, pharmacological properties and analysis. Curr Pharm Anal 5(1): 47-68, 2009. DOI: $10.2174 / 157341209787314936$

33 Futuro DO, Ferreira PG, Nicoletti CD, Borba-Santos LP, Silva FCDA, Rozental S and Ferreira VF: The antifungal activity of naphthoquinones: An integrative review. An Acad Bras Cienc 90(1 Suppl 2): 1187-1214, 2018. PMID: 29873671. DOI: 10.1590/0001-3765201820170815

34 Wellington KW: Understanding cancer and the anticancer activities of naphthoquinones - a review. RSC Adv 5(26): 20309-20338, 2015. DOI: 10.1039/C4RA13547D

35 Tandon VK and Kumar S: Recent development on naphthoquinone derivatives and their therapeutic applications as anticancer agents. Expert Opin Ther Pat 23(9): 1087-1108, 2013. PMID: 23651032. DOI: $10.1517 / 13543776.2013 .798303$

36 Pinto AV and de Castro SL: The trypanocidal activity of naphthoquinones: A review. Molecules 14(11): 4570-4590, 2009. PMID: 19924086. DOI: 10.3390/molecules 14114570

37 Salas CO, Faúndez M, Morello A, Maya JD and Tapia RA: Natural and synthetic naphthoquinones active against trypanosoma cruzi: An initial step towards new drugs for chagas disease. Curr Med Chem 18(1): 144-161, 2011. PMID: 21110810.

38 Asche C: Antitumour quinones. Mini Rev Med Chem 5(5): 449467, 2005. PMID: 15892687.

39 da Rocha DR, de Souza ACG, Resende JALC, Santos WC, dos Santos EA, Pessoa C, de Moraes MO, Costa-Lotufo LV, Montenegro RC and Ferreira VF: Synthesis of new 9hydroxy- $\alpha$ - and 7-hydroxy- $\beta$-pyran naphthoquinones and cytotoxicity against cancer cell lines. Org Biomol Chem 9(11): 4315-4322, 2011. PMID: 21487631. DOI: 10.1039/ c1ob05209h

40 Pérez-Sacau E, Díaz-Peñate RG, Estévez-Braun A, Ravelo AG, García-Castellano JM, Pardo L and Campillo M: Synthesis and pharmacophore modeling of naphthoquinone derivatives with cytotoxic activity in human promyelocytic leukemia hl-60 cell line. J Med Chem 50(4): 696-706, 2007. PMID: 17249647. DOI: $10.1021 / \mathrm{jm} 060849 \mathrm{~b}$

41 Liu KKC, Li J and Sakya S: Synthetic approaches to the 2003 new drugs. Mini Rev Med Chem 4(10): 1105-1125, 2004. PMID: 15579116.
42 Hallak M, Thakur BK, Winn T, Shpilberg O, Bittner S, Granot Y, Levy I and Nathan I: Induction of death of leukemia cells by tw-74, a novel derivative of chloro-naphthoquinone. Anticancer Res 33(1): 183-190, 2013. PMID: 23267144.

43 Sliwoski G, Kothiwale S, Meiler J and Lowe EW: Computational methods in drug discovery. Pharmacol Rev 66(1): 334-395, 2014. PMID: 24381236. DOI: 10.1124/pr.112.007336

44 Rehan M: An anti-cancer drug candidate OSI-027 and its analog as inhibitors of mtor: Computational insights into the inhibitory mechanisms. J Cell Biochem 118(12): 4558-4567, 2017. PMID: 28475291. DOI: $10.1002 /$ jcb.26117

45 Rehan M: A structural insight into the inhibitory mechanism of an orally active pi3k/mtor dual inhibitor, PKI-179 using computational approaches. J Mol Graph Model 62: 226-234, 2015. PMID: 26500112. DOI: 10.1016/j.jmgm.2015.10.005

46 Rehan M, Beg MA, Parveen S, Damanhouri GA and Zaher GF: Computational insights into the inhibitory mechanism of human AKT1 by an orally active inhibitor, MK-2206. PLoS One 9(10): e109705, 2014. PMID: 25329478. DOI: 10.1371/journal. pone. 0109705

47 Jamal MS, Parveen S, Beg MA, Suhail M, Chaudhary AGA, Damanhouri GA, Abuzenadah AM and Rehan M: Anticancer compound plumbagin and its molecular targets: A structural insight into the inhibitory mechanisms using computational approaches. PLoS One 9(2): e87309, 2014. PMID: 24586269. DOI: 10.1371/journal.pone.0087309

48 Batool S, Nawaz MS, Greig NH, Rehan M and Kamal MA: Molecular interaction study of N1-P-fluorobenzyl-cymserine with TNF- $\alpha$, p38 kinase and jnk kinase. Antiinflamm Antiallergy Agents Med Chem 12(2): 129-135, 2013. PMID: 23360257.

49 Rehan M, Ahmad E, Sheikh IA, Abuzenadah AM, Damanhouri GA, Bajouh OS, AlBasri SF, Assiri MM and Beg MA: Androgen and progesterone receptors are targets for bisphenol a (BPA), 4methyl-2,4-bis-(p-hydroxyphenyl)pent-1-ene--a potent metabolite of bpa, and 4-tert-octylphenol: A computational insight. PLoS One 10(9): e0138438, 2015. PMID: 26379041. DOI: 10.1371/journal. pone. 0138438

50 Ahmad A, Ahmad A, Varshney H, Rauf A, Rehan M, Subbarao $\mathrm{N}$ and Khan AU: Designing and synthesis of novel antimicrobial heterocyclic analogs of fatty acids. Eur J Med Chem 70: 887900, 2013. PMID: 24262381. DOI: 10.1016/j.ejmech. 2013.10.051

51 Ewing TJ, Makino S, Skillman AG and Kuntz ID: Dock 4.0: Search strategies for automated molecular docking of flexible molecule databases. J Comput Aided Mol Des 15(5): 411-428, 2001. PMID: 11394736.

52 Pettersen EF, Goddard TD, Huang CC, Couch GS, Greenblatt DM, Meng EC and Ferrin TE: Ucsf chimera - a visualization system for exploratory research and analysis. J Comput Chem 25(13): 1605-1612, 2004. PMID: 15264254. DOI: 10.1002/ jcc.20084

53 DeLano WL: PyMOL. San Carlos, CA. DeLano Scientific, 2002. 54 Laskowski RA and Swindells MB: Ligplot+: Multiple ligandprotein interaction diagrams for drug discovery. J Chem Inf Model 51(10): 2778-2786, 2011. PMID: 21919503. DOI: $10.1021 / \mathrm{ci} 200227 \mathrm{u}$

55 Wang R, Lai L and Wang S: Further development and validation of empirical scoring functions for structure-based binding affinity prediction. J Comput Aided Mol Des 16(1): 11-26, 2002. PMID: 12197663. 
56 Lipinski CA, Lombardo F, Dominy BW and Feeney PJ: Experimental and computational approaches to estimate solubility and permeability in drug discovery and development settings. Adv Drug Deliv Rev 46(1-3): 3-26, 2001. PMID: 11259830.

57 Rehan M and Bajouh OS: Virtual screening of naphthoquinone analogs for potent inhibitors against the cancer-signaling pi3k/akt/mtor pathway. J Cell Biochem, 2018. PMID: 30298630. DOI: $10.1002 /$ jcb. 27100

58 Wang Y, Xiao J, Suzek TO, Zhang J, Wang J, Zhou Z, Han L, Karapetyan K, Dracheva S, Shoemaker BA, Bolton E, Gindulyte A and Bryant SH: Pubchem's bioassay database. Nucleic Acids Res 40(Database issue): D400-412, 2012. PMID: 22140110. DOI: $10.1093 /$ nar/gkr1132

59 National Center for Biotechnology Information. PubChem BioAssay Database; AID=29. https://pubchem.ncbi.nlm.nih.gov/ bioassay/29

60 National Center for Biotechnology Information. PubChem BioAssay Database; AID=67. https://pubchem.ncbi.nlm.nih.gov/ bioassay/67

61 National Center for Biotechnology Information. PubChem BioAssay Database; AID=119. https://pubchem.ncbi.nlm.nih. gov/bioassay/119

62 National Center for Biotechnology Information. PubChem BioAssay Database; AID=125. https://pubchem.ncbi.nlm.nih. gov/bioassay/125

63 National Center for Biotechnology Information. PubChem BioAssay Database; AID=155. https://pubchem.ncbi.nlm. nih.gov/bioassay/155

64 National Center for Biotechnology Information. PubChem BioAssay Database; AID=157. https://pubchem.ncbi.nlm. nih.gov/bioassay/157
65 National Center for Biotechnology Information. PubChem BioAssay Database; AID=161. https://pubchem.ncbi.nlm. nih.gov/bioassay/161

66 National Center for Biotechnology Information. PubChem BioAssay Database; $\mathrm{AID}=165$. https://pubchem.ncbi. nlm.nih.gov/bioassay/165

67 National Center for Biotechnology Information. PubChem BioAssay Database; AID=167. https://pubchem.ncbi. nlm.nih. gov/bioassay/167

68 National Center for Biotechnology Information. PubChem BioAssay Database; AID=175. https://pubchem.ncbi.nlm. nih.gov/bioassay/175

69 National Center for Biotechnology Information. PubChem BioAssay Database; AID=179. https://pubchem.ncbi.nlm. nih.gov/bioassay/179

70 National Center for Biotechnology Information. PubChem BioAssay Database; AID=330. https://pubchem.ncbi.nlm.nih. gov/bioassay $/ 330$

71 National Center for Biotechnology Information. PubChem BioAssay Database; AID=651550. https://pubchem.ncbi.nlm.nih. gov/bioassay/651550

72 National Center for Biotechnology Information. PubChem BioAssay Database; AID=735529. https://pubchem.ncbi.nlm.nih. gov/bioassay/735529

Received March 24, 2019

Revised May 10, 2019

Accepted May 13, 2019 